\title{
Structural instability in an autophosphorylating kinase switch
}

\author{
Michael Grinfeld \\ Steven D. Webb \\ Department of Mathematics, University of Strathclyde, 26 Richmond Street, \\ Glasgow G1 1XH, U.K.
}

\begin{abstract}
We analyse a simple kinase model that exhibits bistability when there is no protein turnover, and show analytically that the property of being bistable is not necessarily conserved when degradation and synthesis of the kinase are taken into account.
\end{abstract}

Key words: Bistability, protein turnover, structural instability

\section{Introduction}

Multistability is an important characteristic of many biological systems; it expresses their ability to act as switches given an appropriate stimulus. More precisely, by multistability we mean a situation in which a stimulus can lead to more than one observable outcome. The outcome may depend on the initial conditions, that is, the state of the system when the stimulus is applied, or more generally on the entire past history of the system. Accounting for multistability is an important necessary step when one wants to understand why in an isogenic, genetically homogeneous population subject to an interference such as ionising radiation, different cells react differently.

The literature on detecting multistability or the lack thereof in networks, and in particular in signal transduction pathways, is growing apace. The reader is referred, for example, to the work of Feinberg et al. [1] or of Sontag and his

Email addresses: michael@maths.strath.ac.uk (Michael Grinfeld), sdw@maths.strath.ac.uk (Steven D. Webb). 
collaborators [2-4], much of it based on the theory of monotone dynamical systems [5] and graph-theoretical methods.

In this paper we will discuss a very simple system that exhibits multistability (in fact, bistability). This is the autophosphorylating kinase example due to Lisman [6], in which much can be done explicitly. The paper of Lisman is very well known but his analysis is incomplete. In particular, very surprisingly, this model, the derivation of which uses only the rules of enzyme kinetics, turns out not to be structurally stable in the following sense: if kinase turnover is incorporated into the equations, bistability can disappear for arbitrarily small values of kinetic constants describing kinase turnover. Observe, in this context, that the models of [2] and [1] do not take protein turnover into account.

Note that autophosphorylating kinases exist and carry out many important tasks. One example is provided by ATM, a DNA damage sensor [9], another, by the calciūm/calmodulin-dependent protein kinase II (CaMKII), which is considered, for instance, in [7]. In that paper, the fact that protein turnover causes the switch to reset itself due to the disappearance of bistability, is clearly acknowledged.

Another paper that deals with this structural instability phenomenon is [10]. There, the authors also suggest a mechanism for enlarging the region of parameter space where bistability persists on addition of protein turnover; in the present paper we show analytically that indeed this mechanism, cooperative stability, will have the effect suggested for it in [10]. We also comment on the lack of cooperative stability in ATM.

Everywhere below we will be using the following convention: $S$ is the concentration (moles per unit volume) of a species $\mathrm{S} ; S_{\text {eq }}$ will denote the equilibrium concentration of S.

\section{The Lisman Model}

Lisman [6] considers the question of stable information storage by unstable molecules. He suggests a switch comprised of a protein kinase-kinase (KK) and a protein kinase, which can activate itself by autophosphorylation. This means that an activated, phosphorylated molecule of the kinase, $\mathrm{K}^{*}$, can phosphorylate an inactive non-phosphorylated molecule $\mathrm{K}$ to create two molecules of $\mathrm{K}^{*}$; see Figure 1.

With reference to Figure 1, we assume that the kinase-kinase has already stimulated the kinase (which Lisman calls kinase-1) and so we have a certain amount of activated phosphorylated kinase $\mathrm{K}^{*}$. Lisman [6, p. 3056] says 


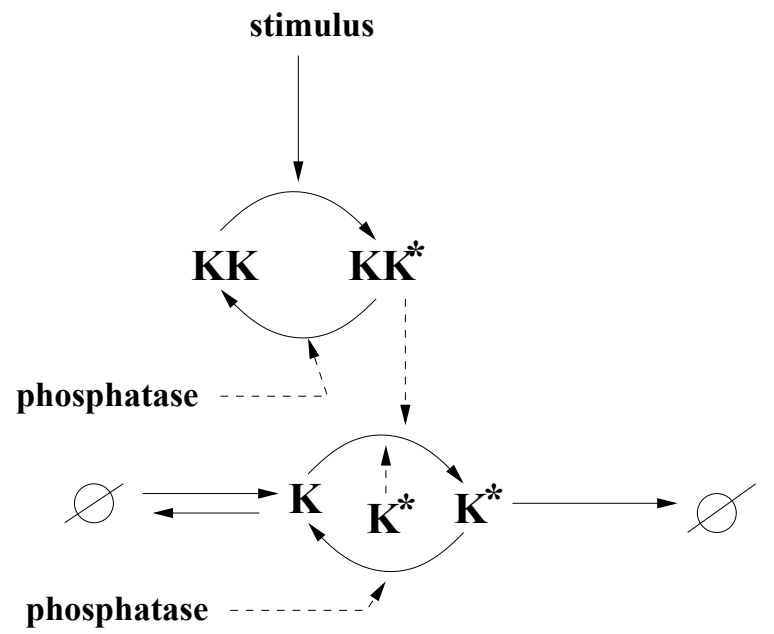

Fig. 1. The Lisman example: dotted arrows indicate catalysis; arrows ending in $\emptyset$ denote degradation; arrow that originates in $\emptyset$ denotes de novo synthesis.

that if the level of the activated kinase is high enough, we have created a dynamic switch, which "therefore will not be reset by reasonable levels of protein turnover. If the switch is on, and kinase- 1 is is active, newly synthesized kinase-1 molecules will become activated, thus replacing active kinase-1 molecules destroyed by protein turnover." Here we investigate this suggestion in detail.

Let us consider the minimal set of processes that operate here.

1. A molecule of $\mathrm{K}$ combines with a molecule of $\mathrm{K}^{*}$ to give two molecules of $\mathrm{K}^{*}$; this is the autophosphorylation step: $K+K^{*} \longrightarrow 2 K^{*}$;

2. A molecule of $\mathrm{K}^{*}$ combines with a molecule of phosphatase, $\mathrm{P}$, to give a molecule of phosphatase and a molecule of $\mathrm{K}: K^{*}+P \longrightarrow K+P$;

3. $\mathrm{K}$ is destroyed (kinase turnover): $K \longrightarrow \emptyset$;

4. $\mathrm{K}^{*}$ is destroyed (kinase turnover): $K^{*} \longrightarrow \emptyset$;

5. $\mathrm{K}$ is created de novo: $\emptyset \longrightarrow K$.

Thus in particular we are making the assumption that the amount of phosphatase is constant in time and that we no longer require the kinase-kinase $\mathrm{KK}^{*}$ to activate the kinase $\mathrm{K}$. To translate this list of reactions into an analysable mathematical model, we must be more explicit about reaction mechanisms.

(a) For autophosphorylation we assume the following scheme:

$$
\mathrm{K}+\mathrm{K}^{*} \underset{b_{1}}{\stackrel{f_{1}}{\rightleftharpoons}} \mathrm{C}_{\mathrm{K}} \stackrel{f_{2}}{\longrightarrow} 2 \mathrm{~K}^{*}
$$

This is the simplest possible mechanism that is in accordance with the rules of 
enzymes kinetics: $\mathrm{K}$ and $\mathrm{K}^{*}$ create a complex, $\mathrm{C}_{\mathrm{K}}$, at the (forward) rate $f_{1}$; this complex is either broken back down to its original constituents at rate $b_{1}$ or proceeds at rate $f_{2}$ (which takes into account the ambient ATP concentration) to phosphorylate the molecule of $\mathrm{K}$ and thus create 2 molecules of $\mathrm{K}^{*}$.

(b) Similarly, for dephosphorylation we have

$$
\mathrm{P}+\mathrm{K}^{*} \underset{b_{3}}{\stackrel{f_{3}}{\rightleftharpoons}} \mathrm{C}_{\mathrm{P}} \stackrel{f_{4}}{\longrightarrow} \mathrm{P}+\mathrm{K} .
$$

(c) For protein degradation we shall assume first order kinetics, that is, that $\mathrm{K}$ is degraded at a rate proportional to its amount with a rate constant $d$ and, similarly that $\mathrm{K}^{*}$ is degraded at a rate proportional to its amount with a rate constant $d^{*}$. We will see that, as also pointed out in [10], the ratio $d^{*} / d$ has an important influence on bistability.

(d) Finally, we assume that $\mathrm{K}$ is created at constant rate $\alpha$ (zero order kinetics). In other words, we are assuming that there is no feedback between its concentration and the rate of its synthesis.

\section{The Governing ODEs}

Using the laws of mass action kinetics, which of course are only valid when the numbers of molecules involved are large and the system is well-stirred, we get the following system of equations:

$$
\begin{aligned}
\frac{d K}{d t}=\alpha-d K- & f_{1} K K^{*}+b_{1} C_{\mathrm{K}}+f_{4} C_{\mathrm{P}}, \\
\frac{d K^{*}}{d t}=-d^{*} K^{*}- & f_{1} K K^{*}+b_{1} C_{\mathrm{K}}+2 f_{2} C_{\mathrm{K}} \\
& \quad-f_{3} K^{*}\left(P_{t o t}-C_{\mathrm{P}}\right)+b_{3} C_{\mathrm{P}}, \\
\frac{d C_{\mathrm{P}}}{d t}= & f_{3} K^{*}\left(P_{t o t}-C_{\mathrm{P}}\right)-\left(b_{3}+f_{4}\right) C_{\mathrm{P}}, \\
\frac{d C_{\mathrm{K}}}{d t}= & f_{1} K K^{*}-\left(b_{1}+f_{2}\right) C_{\mathrm{K}} .
\end{aligned}
$$

We also have the conservation law $P+C_{\mathrm{P}}=P_{\text {tot }}$, where $P_{\text {tot }}$ is the total concentration of phosphatase, assumed constant.

At equilibrium, from the last two equations of (1) we obtain

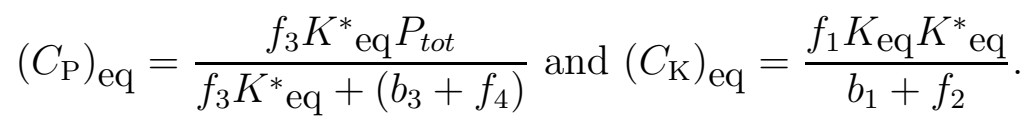


Substituting these formulae into the equations for $d K / d t$ and $d K^{*} / d t$, we have that at equilibrium

$$
\begin{aligned}
& 0=\alpha-d K_{\mathrm{eq}}-\beta K_{\mathrm{eq}} K_{\mathrm{eq}}^{*}+\frac{V_{\max } K^{*} \mathrm{eq}}{K_{\mathrm{eq}}^{*}+K_{M}}, \\
& 0=-d^{*} K^{*} \mathrm{eq}+\beta K_{\mathrm{eq}} K_{\mathrm{eq}}^{*}-\frac{V_{\max } K^{*} \mathrm{eq}}{K^{*} \mathrm{eq}+K_{M}}
\end{aligned}
$$

Here we have put

$$
\beta=\frac{f_{1} f_{2}}{b_{1}+f_{2}}, V_{\max }=f_{4} P_{\text {tot }} \text { and } K_{M}=\frac{b_{3}+f_{4}}{f_{3}} .
$$

These parameters are easy to interpret: $\beta$ measures the strength of autophosphorylation; $V_{\max }$ is the maximal speed of dephosphorylation by the phosphatase, and $K_{M}$, the Michaelis-Menten constant, is the concentration $K^{*}$ for which the phosphatase is working at half its maximal speed $V_{\max }$; thus it measures the avidity of phosphatase for $\mathrm{K}^{*}$.

\section{The Case of No Kinase Turnover}

In this situation in (3) we have $\alpha=0, d=0$ and $d^{*}=0$. Lisman's strategy is to analyse this case and then to suggest that if the turnover parameters are sufficiently small, the behaviour of the system will not change by much.

In the case of zero turnover parameters, the two equations of (3) are identical; $K^{*}$ eq is always a solution. We need to use the additional conservation law,

$$
\frac{d}{d t}\left(K+K^{*}+2 C_{K}+C_{P}\right)=0
$$

Let us call the total concentration of kinase $T$, and so without kinase turnover for all time $T(t)$ is constant, and in particular

$$
\begin{aligned}
& K_{\mathrm{eq}}+K^{*} \text { eq }+2\left(C_{K}\right)_{\mathrm{eq}}+\left(C_{P}\right)_{\mathrm{eq}}=T= \\
& K(0)+K^{*}(0)+2 C_{K}(0)+C_{P}(0) .
\end{aligned}
$$

Thus, putting $K_{\text {eq }}=T-K^{*}$ eq $-2\left(C_{K}\right)_{\text {eq }}-\left(C_{P}\right)_{\text {eq }}$, with $\left(C_{P}\right)_{\text {eq }}$ and $\left(C_{K}\right)_{\text {eq }}$ given by (2), the study of non-zero equilibria of (3) reduces to that of a single 
equation,

$$
\begin{aligned}
\left(K^{*} \text { eq }\right)^{2}+ & \left(\frac{V_{\max }}{f_{4}}+\frac{2 V_{\max }}{f_{2}}-T+K_{M}\right) K_{\mathrm{eq}}^{*} \\
& +\frac{V_{\max }}{\beta}-T K_{M}=0 .
\end{aligned}
$$

Setting $\phi=1+2 f_{4} / f_{2}$, this can be rewritten as

$$
\left(K^{*} \text { eq }\right)^{2}+\left(\phi P_{t o t}-T+K_{M}\right) K^{*} \text { eq }+\frac{V_{\text {max }}}{\beta}-T K_{M}=0 .
$$

This is a quadratic in $K^{*}$ eq, the simplest form of equilibrium equations that can give rise to bistability.

To have positive solutions of (6), we must have

$$
T>T_{0}:=K_{M}+\phi P_{t o t} .
$$

At the same time, we must have

$$
T<\frac{V_{\max }}{\beta K_{M}}
$$

Thus there is an upper and a lower bound on the total concentration of the kinase for which there could be bistability. There is another condition: the discriminant of the quadratic (6) must be positive. This is the condition

$$
\left(\phi P_{t o t}-T+K_{M}\right)^{2}>4\left(\frac{V_{\max }}{\beta}-T K_{M}\right)
$$

Condition (9) says that as the strength of dephosphorylation, as measured by $V_{\max }$, is increased, sooner or later there will be no phosphorylated kinase; similarly, bistability requires sufficient strength of the positive feedback, i.e. autophosphorylation, as measured by $\beta$.

If $\left(V_{\max } / \beta-T_{0} K_{M}\right) \leq 0$, condition (9) is vacuously satisfied. If this is not the case, there is some smallest value $T_{1}>T_{0}$ such that (9) (and thus automatically (7)) holds. Let us put

$$
T_{*}= \begin{cases}T_{1} & \text { if } T_{1} \text { exists } \\ T_{0} & \text { otherwise }\end{cases}
$$


Then all the three conditions can be combined to give the following necessary and sufficient condition for bistability:

$$
T \in I:=\left(T_{*}, \frac{V_{\max }}{\beta K_{M}}\right) .
$$

Note that everything here has the dimensions of concentration. Thus if the interval $I$ in (10) is empty, or if $T$ is not in that interval, there is no bistability in (1).

When the interval in (10) is non-empty, using $T$ as the bifurcation parameter, a typical bifurcation diagram is as in Figure 2: as $T$ is increased, bistability, i.e. the existence two non-negative stable steady states appears by a saddle-node bifurcation and disappears in a transcritical bifurcation, leaving only the high $K^{*}$ state. In that Figure, solid lines correspond to stable solutions.

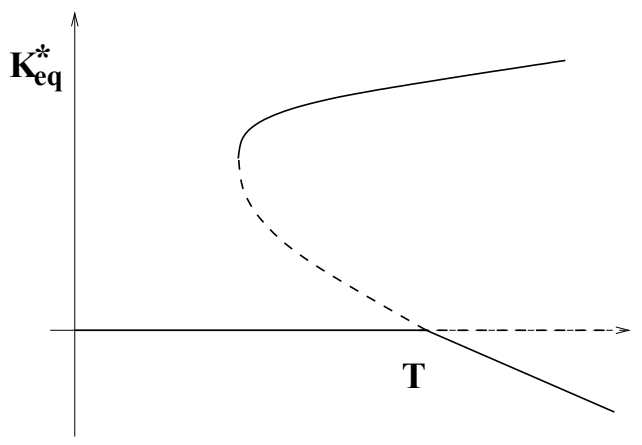

Fig. 2. Bifurcation diagram for the Lisman example

To summarise, bistability in (1) without kinase turnover will occur if only if the initial data $\left\{K(0), K^{*}(0), C_{K}(0), C_{P}(0)\right\}$ satisfies $K(0)+K^{*}(0)+2 C_{K}(0)+$ $C_{P}(0) \in I$.

\section{The Case of Non-zero Kinase Turnover}

In this case, note that if some, but not all, of $\alpha, d, d^{*}$ are non-zero, bistability is impossible, as

$$
\frac{d}{d t}\left(K+K^{*}+C_{P}+2 C_{K}\right)=\alpha-d K-d^{*} K^{*}
$$

So, at equilibrium we have

$$
\alpha-d K_{\mathrm{eq}}-d^{*} K^{*} \text { eq }=0
$$


and thus, for example, if $d=0, K^{*}$ eq is uniquely defined: $K^{*}$ eq $=\alpha / d^{*}$. If all four parameters are non-zero, it is certainly possible to have bistability. In Figure 3 we plot the equilibrium values of $C_{K}, C_{P}, K$ and $K^{*}$ as we vary $d=d^{*}$. Note the trivial branch $C_{K \text { eq }}=C_{P \text { eq }}=K^{*}$ eq $=0, K_{\text {eq }}=\alpha / d$.

(a)

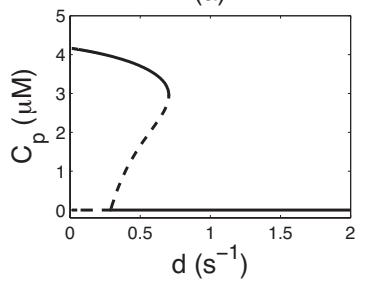

(c)

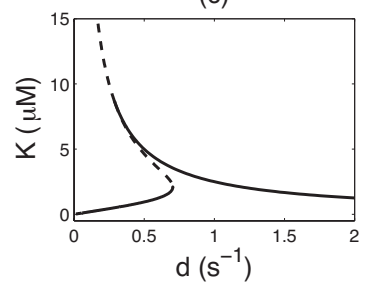

(b)

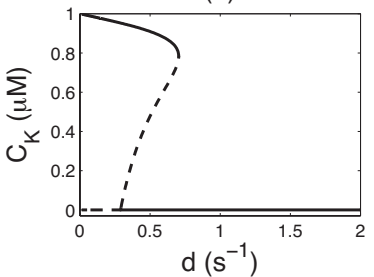

(d)

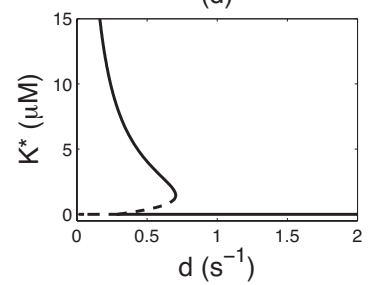

Fig. 3. One-parameter bifurcation diagrams in the Lisman example with protein turnover. $b_{1}=b_{3}=0.1 \mathrm{~s}^{-1}$ and all the other required parameters are taken from the experiments on CaMKII reported in $[7,8], \alpha=2.5 \mathrm{~s}^{-1} \mu \mathrm{M}, f_{1}=3 \mathrm{~s}^{-1} \mu \mathrm{M}, f_{2}=3 \mathrm{~s}^{-1}$, $f_{3}=40 \mathrm{~s}^{-1} \mu \mathrm{M}, f_{4}=10 \mathrm{~s}^{-1}, P_{t o t}=1 \mu \mathrm{M}$. Solid (dashed) curves indicate stable (unstable) branches.

In Figure 4 we present the results of a two-parameter continuation of equilibria in the $(d, \alpha)$ plane, all the rest of the parameters being as in Figure 3.

As we can see from Figure 4, bistability persists only in a wedge in the $(d, \alpha)$ plane. We will now study this phenomenon in more detail. As the full algebra with all three of these parameters being non-zero is cumbersome, we will only deal with two informative particular cases.

(1) We want to understand what is happening when the turnover parameters are "small". For that we need to non-dimensionalise the governing equilibrium equations (3). Setting

$$
\widetilde{K}_{\mathrm{eq}}=K_{M} K_{\mathrm{eq}}, \quad \widetilde{K}_{\mathrm{eq}}^{*}=K_{M} K^{*} \text { eq }, \quad \widetilde{\beta}=\beta K_{M}^{2} / V_{\mathrm{max}}
$$

and

$$
\widetilde{d}=d K_{M} / V_{\max }, \quad \widetilde{d}^{*}=d^{*} K_{M} / V_{\max }, \quad \widetilde{\alpha}=\alpha / V_{\max }
$$



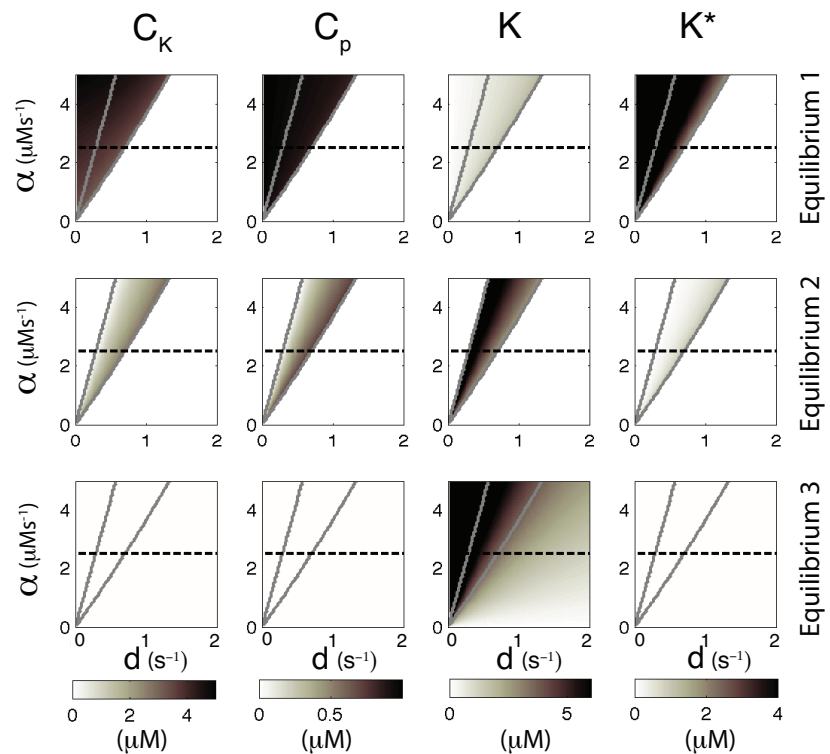

Fig. 4. A two-parameter equilibria continuation in the $(d, \alpha)$ plane. The solid curves denote the boundary of the bistability region and the shading indicates the equilibrium values for $C_{K}$ (left), $C_{P}$ (left-middle), $K$ (right-middle) and $K^{*}$ (right). White denotes the regions where equilibria either do not exist or are zero. The slices through $\alpha=2.5$ as in Figure 3 are indicated by the dashed line.

we have the non-dimensionalised equivalent of (3),

$$
\begin{aligned}
& 0=\widetilde{\alpha}-\widetilde{d K_{\mathrm{eq}}}-\widetilde{\beta} \widetilde{K}_{\mathrm{eq}} \widetilde{K}_{\mathrm{eq}}^{*}+\frac{\widetilde{K}_{\mathrm{eq}}^{*}}{\widetilde{K}_{\mathrm{eq}}^{*}+1}, \\
& 0=-\widetilde{d}^{*} \widetilde{K}_{\mathrm{eq}}^{*}+\widetilde{\beta} \widetilde{K}_{\mathrm{eq}} \widetilde{K}_{\mathrm{eq}}^{*}-\frac{\widetilde{K}_{\mathrm{eq}}^{*}}{\widetilde{K}_{\mathrm{eq}}^{*}+1} .
\end{aligned}
$$

Now let all the three parameters $\widetilde{\alpha}, \tilde{d}, \widetilde{d}^{*}$ be $O(\epsilon)$, for some small $\epsilon>0$ while the ratios $\gamma:=\widetilde{d}^{*} / \widetilde{d}$ and $\widetilde{\alpha} / \widetilde{d}^{*}$ are $O(1)$. Performing on (3) an analysis similar to that in section 4 , we have, in the original variables, the following criterion: there is bistability iff

$$
\frac{\alpha}{d^{*}} \in J:=\left(\max \left(K_{M}, 2 \sqrt{\frac{V_{\max }}{\gamma \beta}}-K_{M}\right), \frac{V_{\max }}{\gamma \beta K_{M}}\right)
$$

to $O(\epsilon)$. Note that it is the ratio $\alpha / d^{*}$ that has the units of concentration, so that something like (13) had to be expected.

For example, if we set $\gamma=1$, i.e. $d=d^{*}, K_{M}=1, \beta=1, V_{\max }=4$, to have bistability, $\alpha / d$ should be in the interval $(3,4)$ to order $\epsilon$. In other words, there is a wedge in the $(d, \alpha)$-plane where bistability occurs. Compare this with Figure 4(a) of [10]. Also note that one of the main points of that 
paper is reflected in (13). The connection is as follows. In [10, p.9563], the authors suggest that to increase the "wedge" in the turnover parameter space where bistability occurs, it is a good idea to make the activated species less susceptible to degradation, for example, by oligomerisation, which enhances thermal stability of the subunits and sequesters sites that are recognisable by proteolytic enzymes. In terms of (13) this corresponds to decreasing $\gamma$ : since the lower bound grows as $1 / \sqrt{\gamma}$ and the upper one as $1 / \gamma$, the interval of ratios $\alpha / d^{*}$ for which there is bistability, increases.

A numerical example of the shape of the bistability wedge using the parameters of Figure 3 is presented in Figure 5(a). The asymptotic results of (13) in this case predict bistability for $\alpha / d \in(3.30784,8.5258)$. As before, the lower curve is of saddle-node bifurcations and the upper one is of transcritical ones.

(a)

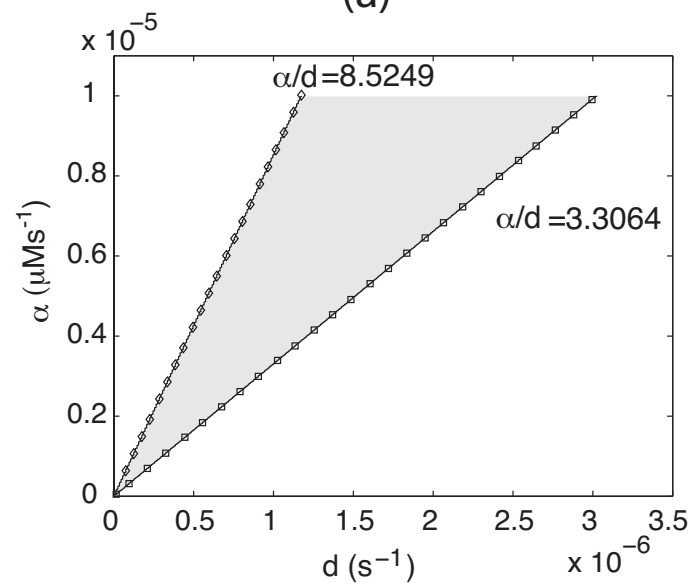

(b)

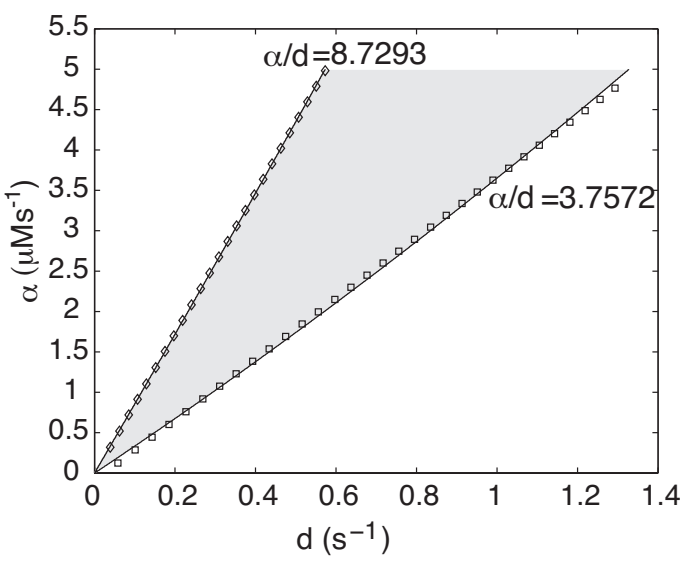

Fig. 5. The bistability wedge in $(d, \alpha)$ plane (a) when $d$ and $\alpha$ are $O\left(10^{-5}\right)$ and (b) when $d$ and $\alpha$ are $O(1)$. The symbols indicate best linear fits with the boundary curves of the bistability region. All other parameters are as in Figure 3.

In Figure 5(b) we show that the asymptotic estimate $\alpha / d \in(3.30784,8.5258)$ is in reasonably good agreement with the numerics even if $\alpha, d$ are not particularly small.

(2) For the particular set of parameters $\gamma=1, \widetilde{\beta}=1 / 4$ mentioned above, one does not need to restrict the parameters $\widetilde{\alpha}$ and $\widetilde{d}$ to be small (here the discriminant of the quadratic factors out) to show that that in the $(d, \alpha)$-plane bistability occurs if

$$
4 \frac{K_{M}^{2}}{V_{\max }} d^{2}+3 K_{M} d<\alpha<4 \frac{K_{M}^{2}}{V_{\max }} d^{2}+4 K_{M} d
$$

which is of course consistent with our asymptotics and again can be compared with Figure 4(a) of [10]. 


\section{Conclusions and Comments}

Equation (13) shows that the system is not structurally stable with respect to bistability: arbitrarily small rates of kinase synthesis and degradation can make bistability (defined as the existence of two positive steady states of (1)) disappear. In other words, suppose that experimentally we cannot detect kinase turnover as $\alpha, d$, and $d^{*}$ are very small. Then by (10), if

$$
K(0)+K^{*}(0)+2 C_{K}(0)+C_{P}(0) \in\left(T_{*}, \frac{V_{\max }}{\beta K_{M}}\right)
$$

we would expect the system to be bistable. However, there is no bistability if there is kinase turnover and $\alpha / d^{*} \notin J$ (see (13)), no matter how small $\alpha, d$, and $d^{*}$ are individually.

This situation raises a number of questions. First of all, how wide-spread is this instability phenomenon; for example, is it also the case in the (conservative) Cdc2/Wee1 system considered in [2]? The second important question is: What additional control structures must be in place in a mass-conserving system so that arbitrarily small variations in protein turnover rates do not destroy bistability; certainly postulating cooperative stability as in [10] does not resolve the problem of structural instability. This question can be rephrased mathematically as follows: what subclass of mass-conserving multistable systems still exhibits multistabilty after the introduction of arbitrary, sufficiently small inflows and outflows?

Note also that our analysis rests on the assumption that synthesis of the kinase $\mathrm{K}$ follows zero order kinetics, while its proteolysis follows first order kinetics. It would be interesting to verify or falsify these assumptions experimentally for example, in the case of CaMKII.

It is not clear a priori that characterisation of bistability in terms of equilibrium states is the right one in biology. For if all the turnover parameters are very small, for finite times the system does behave like the one for which they are zero, i.e. the one in which the total amount of kinase is conserved. So even if there is no bistability as disclosed to us by an analysis of steady states, for finite time the system may maintain a high level of $\mathrm{K}^{*}$, i.e. memory will decay but slowly; see [7] for a discussion of this phenomenon.

Finally, note that in the case of ATM, it is the activated form that is a monomer, while inactive protein is found in dimeric units [9]. This situation would correspond to the case of large $\gamma$ in (13). In the context of DNA damage sensing, unlike the case of memory in the CNS, it is very important to switch ATM off as soon as possible, as it may coopt p53 to sentence the cell to death 
by apoptosis.

\section{Acknowledgements}

We are grateful to anonymous referees who have directed us to [10] and [7] and whose comments have helped us to improve the clarity of exposition.

\section{References}

[1] G. Craciun, Y. Tang, and M. Feinberg, Understanding bistability in complex enzyme-driven reactions, Proc. Nat. Acad. Sci. USA 103 (2006) 8697-8702.

[2] D. Angeli, J. E. Ferrell, Jr., and E. D. Sontag, Detection of multistability, bifurcations and hysteresis in a large class of positive-feedback systems, Proc. Nat. Acad. Sci. USA, 101 (2004) 1822-1827.

[3] D. Angeli, P. de Leenheer, and E. D. Sontag, A graph-theoretical approach to monotonicity and convergence in chemical reaction networks, Preprint, February 2006.

[4] D. Angeli and E. D. Sontag, Multistability in monotone input/output systems, Syst. Control Lett. 51 (2004) 185-202.

[5] H. L. Smith, Monotone Dynamical Systems: Introduction to the Theory of Competitive and Cooperative Systems, AMS, Providence, R. I. 1995.

[6] J. E. Lisman, A mechanism for memory storage insensitive to molecular turnover: a bistable autophosphorylating kinase, Proc. Nat. Acad. Sci. USA, 82 (1985) 3055-3057.

[7] P. Miller, A. M. Zhabotinsky, J. E. Lisman, and X.-J. Wang, The stability of a stochastic CaMKII switch: dependence on the number of enzyme molecules and protein turnover, PLoS Biology 3 (2005) 0705-0717.

[8] A. M. Zhabotinsky, Bistability in the $\mathrm{Ca}^{2+} /$ Calmodium-dependent protein kinase-phosphatase system, Biophys. J. 79 (2000) 2211-2221.

[9] C. J. Bakkenist and M. B. Kastan, DNA damage activates ATM through intermolecular autophosphorylation and dimer dissociation, Nature 421 (2003) 499-506.

[10] N. E. Buchler, U. Gerland, and T. Hwa, Nonlinear protein degradation and the function of genetic circuits, Proc. Nat. Acad. Sci. USA 102 (2005), 9559-9564. 